\title{
Thermoelectric Effect in Photon-Assisted, Spin-Polarized Tunnelling through a Quantum Dot
}

\author{
K. BOCIAN* AND W. RudzIŃSKI \\ Faculty of Physics, Adam Mickiewicz University, Umultowska 85, 61-614 Poznań, Poland
}

\begin{abstract}
Features of electric and thermal conductance, Seebeck coefficient as well as figure of merit in tunnelling through a single level quantum dot connected to external ferromagnetic leads are investigated theoretically using the equation of motion method within the nonequilibrium Green function technique. The influence of the harmonic ac field on the transport characteristics for the considered system is discussed in detail. In particular, it is shown that the photonic field strongly modulates the Seebeck coefficient, thus resulting in multi-peak structure of figure of merit. Thermoelectric phenomenon in a hybrid junction with the dot attached to two ferromagnetic and one superconducting lead is also discussed.
\end{abstract}

DOI: 10.12693/APhysPolA.127.484

PACS: $72.25 . \mathrm{Mk}, 73.23 . \mathrm{Hk}, 73.63 . \mathrm{Kv}, 74.25 . \mathrm{F}-$

\section{Introduction}

Time-dependent tunnelling through low dimensional structures has attracted much attention during the last decade [1]. In particular, such systems offer a possibility to explore new phenomena dealing with photonassisted tunnelling transport. A number of theoretical and experimental works have been devoted to systems based on quantum dots (QD) coupled via tunnelling barriers to ferromagnetic or superconducting electrodes (see e.g. $[2-5]$ ). Souza et al. [2] showed that spin-polarized electrons in the ferromagnet-QD-ferromagnet ( $\mathrm{F}-\mathrm{QD}-\mathrm{F})$ tunnelling junction, can tunnel through inelastic photonassisted transmission channels. Also, it was shown that due to Zeeman splitting of the dot level one may control current polarization by changing gate frequency. In turn, Chi and Dubi [3] studied thermoelectric features of the same system $\mathrm{F}-\mathrm{QD}-\mathrm{F}$, and they predicted that additional tunnelling channels may induce an enhancement of figure of merit. When superconducting electrode is connected to the system the so-called proximity effect occurs and it gives rise to the Andreev resonance picks in the differential conductance. Sun et al. [4] showed that in the system based on QD attached to normal and superconductor leads (N-QD-S), additional conduction channels in the region of superconductor energy gap may appear. It has been shown that due to photon-assisted Andreev tunnelling a series of satellite peaks emerge on both sides of the main resonances of the conductance. In [5] the thermoelectric effect in the N-QD-S system has been studied. Thermal properties of the system in Kondo regime have been investigated for different superconducting gaps and it was predicted that thermopower may be enhanced at temperatures close to the superconducting transition temperature.

* corresponding author; e-mail: bocian@amu.edu.pl
The aim of the present paper is to discuss photonassisted tunneling transport through a quantum dot attached to two ferromagnetic and one superconductor leads. Thus, modifications of thermoelectric characteristics due to interplay between the dot-photon and the dotsuperconductor coupling strengths are analyzed. In particular, we predict that the Andreev transmission occurring in the presence of the harmonic ac photon field gives rise to a significant suppression of the Seebeck coefficient with increasing dot-superconductor coupling strength.

\section{Model and method}

Consider a non-interacting, single-level quantum dot weakly coupled to two ferromagnetic and one superconducting external electrodes (Fig. 1). The system is described by the model Hamiltonian given by $H=$ $\sum_{\nu} H_{F}^{\nu}+H_{S}+H_{Q D}+H_{T}$. The first part of Hamiltonian, $H_{F}^{\nu}=\sum_{k, \sigma} \varepsilon_{\nu k \sigma} c_{\nu k \sigma}^{\dagger} c_{\nu k \sigma}$, describes ferromagnetic electrode (with $\nu=1$ for first and $\nu=2$ for second electrode), where $\varepsilon_{\nu k \sigma}$ is the single-particle energy and $c_{\nu k \sigma}^{\dagger}\left(c_{\nu k \sigma}\right)$ is creation (annihilation) operator for particle with wave vector $k$ and spin $\sigma$ in $\nu$ electrode. The second part is BCS Hamiltonian for superconducting lead, $H_{S}=\sum_{p, \sigma} \varepsilon_{p \sigma} s_{p \sigma}^{\dagger} s_{p \sigma}+\sum_{p}\left(\Delta_{s} s_{-p \uparrow}^{\dagger} s_{p \downarrow}^{\dagger}+\Delta_{s}^{*} s_{p \downarrow} s_{-p \uparrow}\right)$, with $\varepsilon_{p \sigma}$ being single-particle energy with wave vector $p$ and corresponding creation $s_{p \sigma}^{\dagger}$ (annihilation $s_{p \sigma}$ ) operator, $\Delta_{s}$ is superconductor energy gap. Quantum

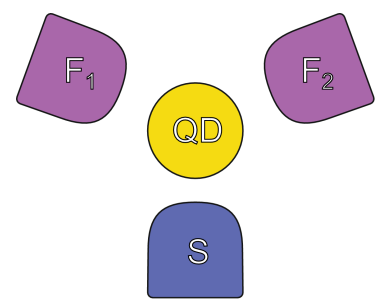

Fig. 1. Schematic of the system. Quantum dot (QD) is coupled via tunnelling barriers to two ferromagnets (F1, F2) and to a superconductor (S). 
dot is described by $H_{Q D}=\varepsilon(t) \sum_{\sigma} d_{\sigma}^{\dagger} d_{\sigma}$ with timedependent level of the dot, $\varepsilon(t)=\varepsilon_{0}+\Delta_{0} \cos \left(\omega_{0} t\right)$ and creation $d_{\sigma}^{\dagger}$ (annihilation $d_{\sigma}$ ) operator. Here, $\Delta_{0}$ describes the strength of the dot-photon field coupling, whereas $\omega_{0}$ stands for photon frequency. The last term of the model Hamiltonian is the tunnelling term, $H_{T}=$ $\sum_{\nu} \sum_{k, \sigma} T_{k \sigma}^{\nu} d_{\sigma}^{\dagger} c_{\nu k \sigma}+\sum_{p, \sigma} T_{p \sigma} d_{\sigma}^{\dagger} s_{p \sigma}+$ H.c., with tunnelling amplitude $T_{k \sigma}^{\nu}\left(T_{p \sigma}\right)$ between $\nu$-th ferromagnetic (superconducting) electrode and the quantum dot.

Using nonequilibrium Green function method and Dyson equation we can determine the time-averaged retarded Green function as (see [1] for details) $G^{R}(\omega)=$ $\sum_{n=-\infty}^{\infty} J_{n}^{2}\left(\frac{\Delta_{0}}{\omega_{0}}\right) G_{n}^{R}(\omega)$, with $J_{n}$ being $n$-th order Bessel function and $\boldsymbol{G}_{n}^{R}(\omega)=\left[\left[\boldsymbol{g}_{0 n}^{R}(\omega)\right]^{-1}-\boldsymbol{\sigma}^{R}(\omega)\right]^{-1}$. Main contribution from ac field is visible in uncoupled dot Green function $\boldsymbol{g}_{0 n}^{R}(\omega)$, where in $4 \times 4$ Nambu representation it takes the form of diagonal matrix with $\left[g_{0 n}^{R}(\omega)\right]^{-1}=\omega \mp \varepsilon_{0} \mp n \omega_{0}$, where minus sign is for $g_{0 n, 11}^{R}(\omega), \quad g_{0 n, 33}^{R}(\omega)$ elements and plus sign is for $g_{0 n, 22}^{R}(\omega), g_{0 n, 44}^{R}(\omega)$ elements of the matrix. In linear response regime thermoelectric properties can be calculated from equa- tions for charge conductance $G=e^{2} \sum_{\sigma} L_{0 \sigma}$, thermal conductance $\kappa=\frac{1}{T}\left[\sum_{\sigma} L_{2 \sigma}-\left(\sum_{\sigma} L_{1 \sigma}\right)^{2} /\left(\sum_{\sigma} L_{0 \sigma}\right)\right]$, Seebeck coefficient $S=-\frac{1}{2 e T} \sum_{\sigma} \frac{L_{1 \sigma}}{L_{0 \sigma}}$ and figure of merit $Z T=\frac{G S^{2} T}{\kappa}$, where coefficients $L_{n \sigma}=$ $-\int \mathrm{d} \omega(\omega-\mu)^{n} \frac{\partial f(\omega)}{\partial \omega} T_{\sigma}(\omega)$ with $\mu$ being chemical potential and $f(\omega)$ Fermi-Dirac distribution. If only an effect of superconducting electrode on thermoelectric transport between ferromagnetic electrodes is to be studied, for the transmission coefficient we take $T_{\sigma}(\omega)=$ $\sum_{n=-\infty}^{\infty} J_{n}^{2}\left(\frac{\Delta_{0}}{\omega_{0}}\right)\left(\boldsymbol{G}_{n}^{R}(\omega) \boldsymbol{\Gamma}_{F}^{1} \boldsymbol{G}_{n}^{A}(\omega) \boldsymbol{\Gamma}_{F}^{2}\right)_{i i}(i i=11$ for $\sigma=\uparrow$ and $i i=33$ for $\sigma=\downarrow)$ with $\boldsymbol{\Gamma}_{F}^{\nu}$ being diagonal coupling matrix between QD and $\nu$-th ferromagnetic electrode with elements $\Gamma_{F \uparrow(\downarrow)}^{\nu}=\Gamma_{f 0}^{\nu}\left(1 \pm P^{\nu}\right)$.

\section{Numerical results}

To investigate thermoelectric properties of the system within the Andreev tunnelling regime, we take for calculations an infinite superconductor energy gap $\Delta \rightarrow \infty$. Moreover, low temperature $k_{B} T=0.001$ is assumed and equilibrium situation $\mu=0$ is taken into account. Ferromagnetic leads are coupled to QD with a fixed strength, $\Gamma_{f 0}=\Gamma_{f 0}^{1}=\Gamma_{f 0}^{2}=0.1$, and the polarization of each electrode is assumed to be $P=0.5$.

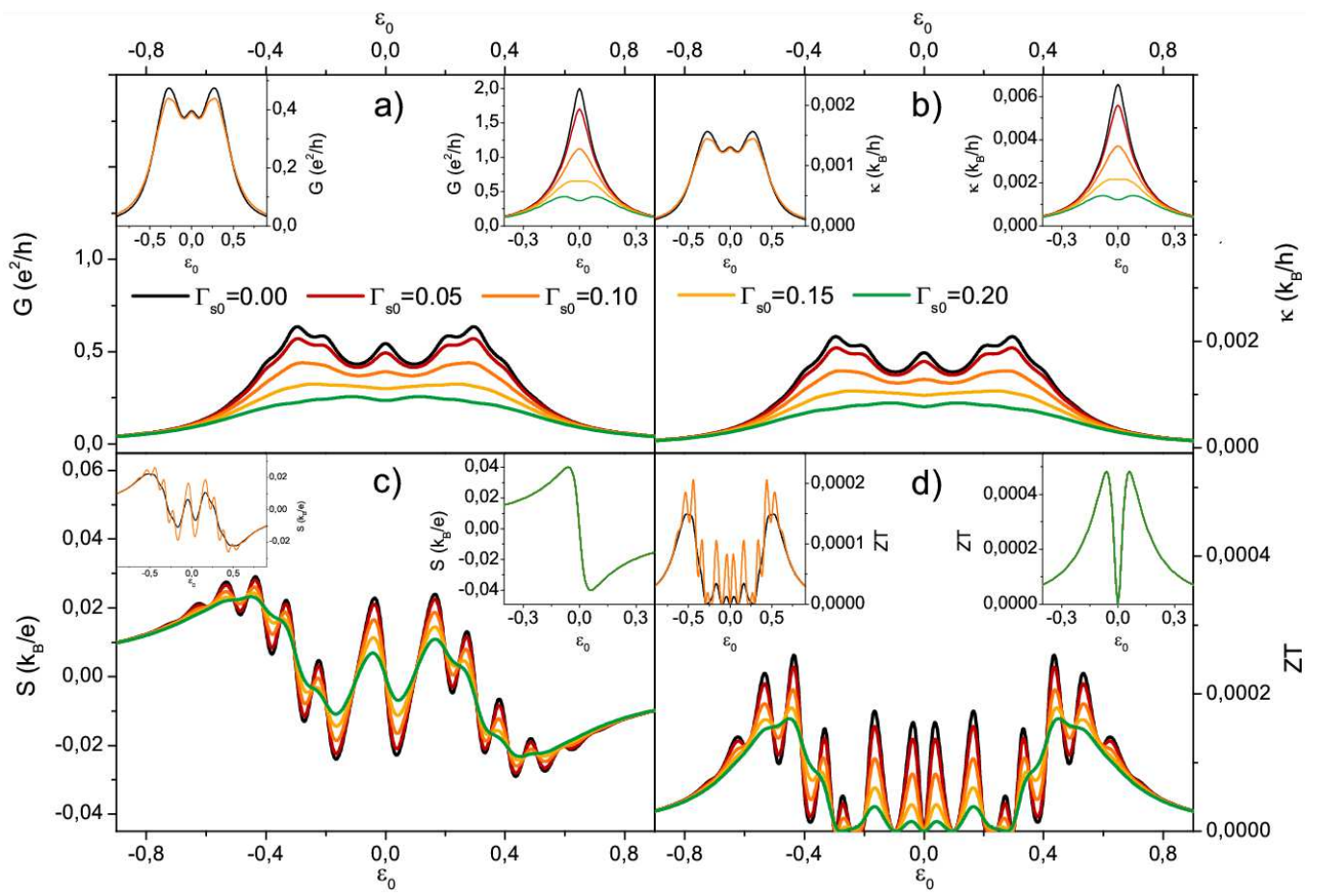

Fig. 2. Electric conductance $G(\mathrm{a})$, thermal conductance $\kappa(\mathrm{b})$, Seebeck coefficient $S$ (c) and figure of merit $Z T$ (d) as a function of dot's level position $\varepsilon_{0}$ for selected values of the QD-S coupling strength parameter $\Gamma_{s 0}$. The parameters describing the ac field are $\omega_{0}=0.1$ and $\Delta_{0}=0.4$. The insets on the right upper side of each a) $-\mathrm{d}$ ) panel show the corresponding characteristics calculated for $\Delta_{0}=0$. The effect of the lead polarization is shown in the insets located on the left upper side of each a)-d) panel, where $G, \kappa, S$ and $Z T$ are shown for the case of $\Gamma_{s 0}=0.1, P=0$ (black lines) and $P=0.5$ (red lines).

Figure 2 shows electric conductance $G$, thermal conductance $\kappa$, Seebeck coefficient $S$ and thermoelectric figure of merit $Z T$ versus dot energy level $\varepsilon_{0}$, obtained for selected values of the quantity $\Gamma_{s 0}$. Consider first the situation without the photon field (see the insets on the right upper side of each panel in Fig. 2). When the su- 


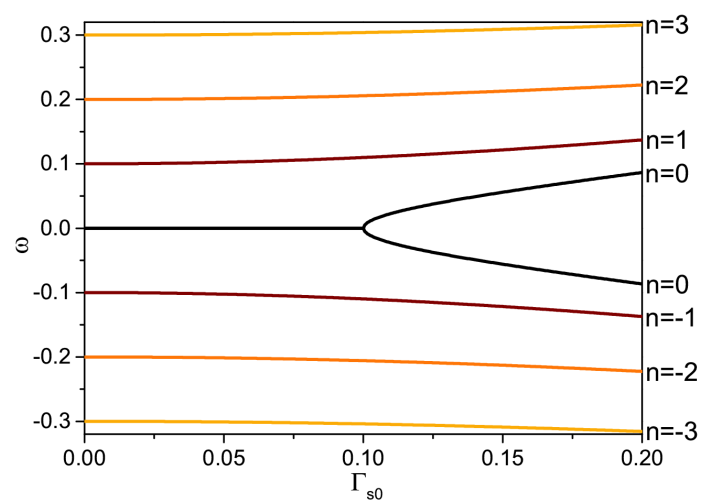

Fig. 3. Position of the main and side resonances in spectral function versus the QD-S coupling strength parameter $\Gamma_{s 0}$. The splitting of the main resonance $(n=0)$ into the two Andreev bound states appears at $\Gamma_{s 0}=\Gamma_{f 0}=0.1$. For increasing $\Gamma_{s 0}$, the satellite resonances are slightly shifted towards higher frequencies.

perconductor lead is uncoupled, $\Gamma_{s 0}=0$, then the charge conductance has one resonance peak at $\varepsilon_{0}=0$ with the maximum value $2 \frac{e^{2}}{h}$. With increasing $\Gamma_{s 0}$ the conductance decreases and for strong enough coupling to superconductor (see the case of $\Gamma_{s 0}>\Gamma_{f 0}$ ) we can distinguish two peaks corresponding to the Andreev resonances localized at $\varepsilon_{0} \approx \pm \frac{\Gamma_{s 0}}{2}$. As clearly seen in Fig. $2 \mathrm{~b}$, thermal conductance follows the behavior of the charge conductance. By contrast, the other thermoelectric characteristics, i.e. the Seebeck coefficient and the figure of merit are insensitive to the variation of the $\Gamma_{s 0}$ parameter (see insets in Fig. 2c, d). For the system without the ac field, thermopower is antisymmetric with respect to the position of the dot level. When dot level is below chemical potential $\mu=0$ mainly holes participate in thermal flow of particles and thermopower stays positive. When crossing chemical potential electrons starts dominate transport what is corresponded to changing sign of thermopower to negative. Thus the figure of merit, being the measure of the thermoelectric efficiency, exhibit the minimum at $\varepsilon_{0}=0$ and two maxima corresponding to maximum values of the Seebeck coefficient.

The thermoelectric features of the system change dramatically if the transmission occurs in the presence of the photon field. First, notice that on both sides of the conductances photon sidebands appear, located at photon energies $\pm n \omega_{0}$. Height of each peak is modulated by square of $n$-th Bessel function $J_{n}^{2}\left(\frac{\Delta_{0}}{\omega_{0}}\right)$. Thus, for the assumed strength of the dot-photon coupling described by $\Delta_{0}$ minima appear at $\varepsilon_{0} \approx \pm \omega_{0}$. The latter property is a photon-driven interference effect. Moreover, as a result of an interplay between the ac field as well as the coupling to the superconductor lead, the conductance suppression is observed.

Second, the photon field gives rise to suppression of thermopower and figure of merit. Simultaneously, the ac field leads to oscillations of these quantities with respect to the position of the dot discrete level. In contrast to the case of $\Delta_{0}=0$, for which the Seebeck coefficient and figure of merit overlap with varying $\Gamma_{s 0}$, our results show that a $\Gamma_{s 0}$-dependent, ac field-induced splitting of $S$ and $Z T$ characteristics may occur. Moreover, a further suppression of thermopower and figure of merit with increasing $\Gamma_{s 0}$ is observed.

Finally, it is found that the QD-S coupling strength gives rise to renormalization of the photon satellite states. It is shown in detail in Fig. 3, where the positions of the main and the first three photon resonances in the spectral function for the system are displayed for a wide range of the $\Gamma_{s 0}$ (and fixed $\Gamma_{f 0}$ ) parameter.

The effect of polarization of the external ferromagnetic leads on the transport properties of the considered hybrid system has been studied, as well (insets on the left upper side of each panel in Fig. 2). It is seen that in the presence of the AR phenomenon the spin-polarized transmission gives rise to suppression of the conductances. Simultaneously, one observes an enhancement of oscillations of the thermopower and $Z T$ with increasing $P$.

\section{Conclusions}

Using nonequilibrium Green function method we have studied thermoelectric phenomenon in a hybrid tunnelling junction based on a single level quantum dot interacting with a harmonic photon field and coupled weakly to two ferromagnetic electrodes and to one superconductor lead. We have shown that the ac field may modulate significantly the thermoelectric characteristics, leading in particular to multi-peak dependencies of the electric and thermal conductance as well as to oscillations of thermopower and the figure of merit. By taking into account the influence of the third, superconducting lead we have found that for transmission in the Andreev tunneling regime one may observe significant suppression of the charge and thermal transport characteristics. Finally, a splitting of thermopower with respect to the QD-S coupling strength has been predicted.

\section{Acknowledgments}

This work was supported by the National Science Center in Poland as the project No. DEC2012/04/A/ST3/00372.

\section{References}

[1] G. Platero, R. Aguado, Phys. Rep. 395, 1 (2004).

[2] F.M. Souza, T.L. Carrara, E. Vernek, Phys. Rev. B 84, 115322 (2011).

[3] F. Chi, Y.Dybi, J. Phys: Condens. Matter 24, 145301 (2012).

[4] Q.F. Sun, J. Wang, T.H. Lin, Phys. Rev. B 59, 13126 (1999).

[5] M. Krawiec, Acta Phys. Pol. A 114, 115 (2008). 